\title{
Propuesta de Clasificación de las Variaciones del Músculo Bíceps Braquial y Estudio en una Muestra de Población Colombiana
}

\author{
Proposal of Classification for the Variations of the Biceps Brachii \\ Muscle and Study in a Sample of Colombian Population
}

\author{
Alian Guerrero'; Gloria Patricia Baena-Caldas ${ }^{2,4}$; Elizabeth Peña²; José Rafael Tovar Cuevas ${ }^{3}$ \& Luz Marina Corrales ${ }^{4,5}$
}

GUERRERO, A.; BAENA-CALdAS, G. P.; PEÑA, E.; TOVAR, C. J. R. \& CORRALES, L. M. Propuesta de clasificación de las variaciones del músculo bíceps braquial y estudio en una muestra de población colombiana. Int. J. Morphol., 36(1):54-58, 2018.

RESUMEN: Las variaciones anatómicas del músculo bíceps braquial en cuanto a la presencia de cabezas accesorias o supernumerarias pueden tener incidencia importante en los diagnósticos clínicos y radiológicos, así como en procedimientos quirúrgicos. La ausencia de una clasificación adecuada para las variaciones del músculo bíceps braquial y los hallazgos del presente estudio le permitieron a los autores presentar una propuesta de clasificación basada en el número de cabezas accesorias y en el origen de ellas. El objetivo del estudio fue determinar la frecuencia con que se presentan variaciones en el número de cabezas accesorias y proponer una clasificación para las variaciones del músculo bíceps braquial. En el estudio de tipo descriptivo, de corte transversal, se analizaron 90 brazos de 46 cadáveres colombianos. Se encontró una alta frecuencia (19,6\%) en las variaciones del músculo, 4,3\% bilateralmente y 15,2 \% unilateralmente. En los 11 brazos (12,2\%) que presentaron la variación del músculo, la presencia de una sola cabeza accesoria fue la más alta $(81,8 \%)$, siendo el origen más frecuente en ellas el clasificado por los autores como 3b, 3 para la ubicación inferior medial y b para el intervalo entre la inserción del músculo coracobraquial y el origen del músculo braquial. Por lo detallado de la clasificación propuesta, se recomienda su uso cuando se reporten casos de variaciones anatómicas del músculo bíceps braquial. En los cadáveres de la población colombiana estudiados se encontró una alta frecuencia para la presencia de cabezas accesorias de este músculo, que amerita ser conocida por el personal médico.

PALABRAS CLAVE: Variación anatómica; Músculo bíceps braquial; Cabezas accesorias; Brazo; Cadáver.

\section{INTRODUCCIÓN}

El músculo bíceps braquial (MBB), ubicado en la región anterior del brazo, presenta dos vientres denominados cabeza corta y cabeza larga. La primera se origina en la parte lateral del ápice del proceso coracoideo y la segunda en el tubérculo supraglenoideo de la escápula; ambas cabezas se unen hacia la parte media del brazo para terminar en un tendón común que se inserta en la tuberosidad del radio y que emite una expansión hacia la fascia del antebrazo denominada aponeurosis bicipital. Es un potente supinador del antebrazo y por su ubicación en la parte anterior de la articulación del codo participa en la flexión del antebrazo, con mayor eficiencia cuando el antebrazo está en supinación. El MBB es inervado por el nervio musculocutáneo, que recibe de los ramos ventrales de los nervios espinales CV, CVI y CVII, y es irrigado y drenado por los vasos braquiales, con una contribución de los vasos circunflejos humerales anteriores (Bergman et al., 1988; Delgado Garcia, 1996).

El MBB puede presentar muchas variaciones (Asvat et al., 1993; Nakatani et al., 1998; Nayak et al., 2008; Ilayperuma et al., 2011; Ballesteros et al., 2014; Duran \& Arquez, 2016). En un estudio realizado en 350 miembros superiores, Rodríguez-Niedenführ et al. (2003) reportan la presencia de una o dos cabezas supernumerarias o accesorias del MBB. Para el caso del músculo con tres cabezas, una de ellas supernumeraria, plantean denominarlas según su ubicación con respecto al origen, en humeral superior, humeral inferomedial y humeral inferolateral, e incluyen otras consideradas como segmentaciones o duplicaciones de las cabezas cortas o de la largas.

\footnotetext{
${ }^{1}$ Médico y Cirujano. Estudiante de Maestría en Ciencias Biomédicas. Facultad de Salud, Universidad del Valle, Cali, Colombia.

${ }^{2}$ Magister en Ciencias Biomédicas. Docente Departamento de Morfología, Facultad de Salud, Universidad del Valle, Cali, Colombia.

${ }^{3} \mathrm{PhD}$ en Estadística. Docente de la Escuela de Estadística, Universidad del Valle, Cali, Colombia.

${ }^{4}$ Docente Programa de Medicina, Facultad de Salud, Universidad Libre, Cali, Colombia.

${ }^{5}$ Docente Facultad de Medicina, Universidad Santiago de Cali, Cali, Colombia.
} 
La presencia de cabezas accesorias puede causar confusión al momento de evaluar imágenes diagnósticas (Gheno et al., 2010; Lutterbach-Penna et al., 2014) o de realizar procedimientos quirúrgicos (Warner et al., 1992). Al respecto, Warner et al. reportaron la presencia de una cabeza accesoria que se originaba en la cápsula de la articulación glenohumeral, la cual, en el momento de la cirugía, debió ser reclinada lateralmente, con el posible riesgo de desgarrar la cápsula articular. Se ha postulado, incluso, el posible papel etiológico de una cabeza accesoria en patologías compresivas de vasos y nervios (Nakatani et al.). Sin embargo, la frecuencia de aparición de estas cabezas accesorias difiere en las muestras tomadas para estudio en diferentes países (Lee, 2013).

Cabe resaltar que aunque en la literatura son frecuentes los reportes de las variaciones anatómicas del MBB en cuanto al número y el origen de cabezas accesorias que este puede presentar, no existe una clasificación adecuada que estandarice dichas variaciones. Por tal motivo, el presente estudio pretende proponer una clasificación para las variaciones anatómicas basada en el número de cabezas accesorias del $\mathrm{MBB}$ encontradas en una muestra de la población colombiana y determinar la frecuencia con que dichas variaciones se presentan.

\section{MATERIAL Y MÉTODO}

Se realizó un estudio descriptivo, observacional de corte transversal, en el que se tomó como población de estudio 90 miembros superiores de 46 cadáveres, 4 femeninos y 42 masculinos (un cadáver presentaba un solo miembro su- perior y en otro no fue posible realizar la disección del brazo), pertenecientes a las salas de disección de las Universidades del Valle, Libre y Santiago de Cali, todas localizadas en la ciudad de Cali, Colombia. Los cadáveres se encontraban conservados con una solución de formalina al $10 \%$ y habían sido disecados por estudiantes del programa de Medicina de cada universidad.

En cada miembro superior de cada cadáver se observó el número de cabezas que presentaba el MBB y el origen e inserción de cada una de ellas; los datos fueron registrados y sometidos a análisis estadístico descriptivo para encontrar la frecuencia con que se presentaban las variaciones anatómicas del músculo en la muestra total y en los subgrupos considerados, los cuales fueron denominados bilateral, unilateral derecho y unilateral izquierdo.

Para la caracterización y análisis de las variaciones encontradas se intentó tomar como base la clasificación propuesta por Rodríguez-Niedenführ et al. la cual atiende al origen de las cabezas supernumerarias como característica de clasificación. Sin embargo, teniendo en cuenta la literatura revisada y las variaciones reportadas por otros autores para el MBB, los autores del presente estudio pueden afirmar que no existe una clasificación formal de éstas. Por lo cual, atendiendo a la clasificación de Rodríguez-Niedenführ et al., a la literatura revisada y a lo observado en el presente estudio, los autores proponen una clasificación basada en el número de cabezas accesorias del MBB y en el sitio de origen de estas cabezas. En primera instancia se clasifican los músculos según el número de cabezas accesorias: Tipo I para una cabeza accesoria, Tipo II para dos cabezas, Tipo III para tres cabezas, y así sucesivamente. Tomando como referencia la posición en que se encuentre la cabeza accesoria en

Tabla I. Propuesta de clasificación de las cabezas accesorias del músculo bíceps braquial (Fuente: autores).

\begin{tabular}{|c|c|}
\hline Ubicación según región del brazo & Origen \\
\hline \multirow[t]{5}{*}{ 1. Superior tejidos óseos } & a. Tubérculo mayor. \\
\hline & b. Tubérculo menor. \\
\hline & c. Fondo del surco intertubercular. \\
\hline & d. Cresta medial del surco intertubercular. \\
\hline & e. Cresta lateral del surco intertubercular. \\
\hline \multirow[t]{3}{*}{ 2. Superior tejidos blandos } & a. Cápsula articular. \\
\hline & b. Margen inferior del músculo pectoral mayor. \\
\hline & c. Superficie anterior del músculo deltoideo. \\
\hline \multirow[t]{5}{*}{ 3. Inferomedial } & a. Músculo coracobraquial. \\
\hline & b. Cara anteromedial del húmero, entre la inserción del músculo \\
\hline & coracobraquial y el origen del músculo braquial. \\
\hline & c. Septo intermuscular medial. \\
\hline & d. Músculo braquial. \\
\hline 4. Infero late ral & $\begin{array}{l}\text { a. Septo intermuscular lateral, entre la inserción del músculo deltoideo } \\
\text { y el origen del músculo braquiorradial. } \\
\text { b. Desde la cresta supracondilar. }\end{array}$ \\
\hline 5. Otras & c. Cabezas accesorias que no cumplen con los criterios acordados. \\
\hline
\end{tabular}



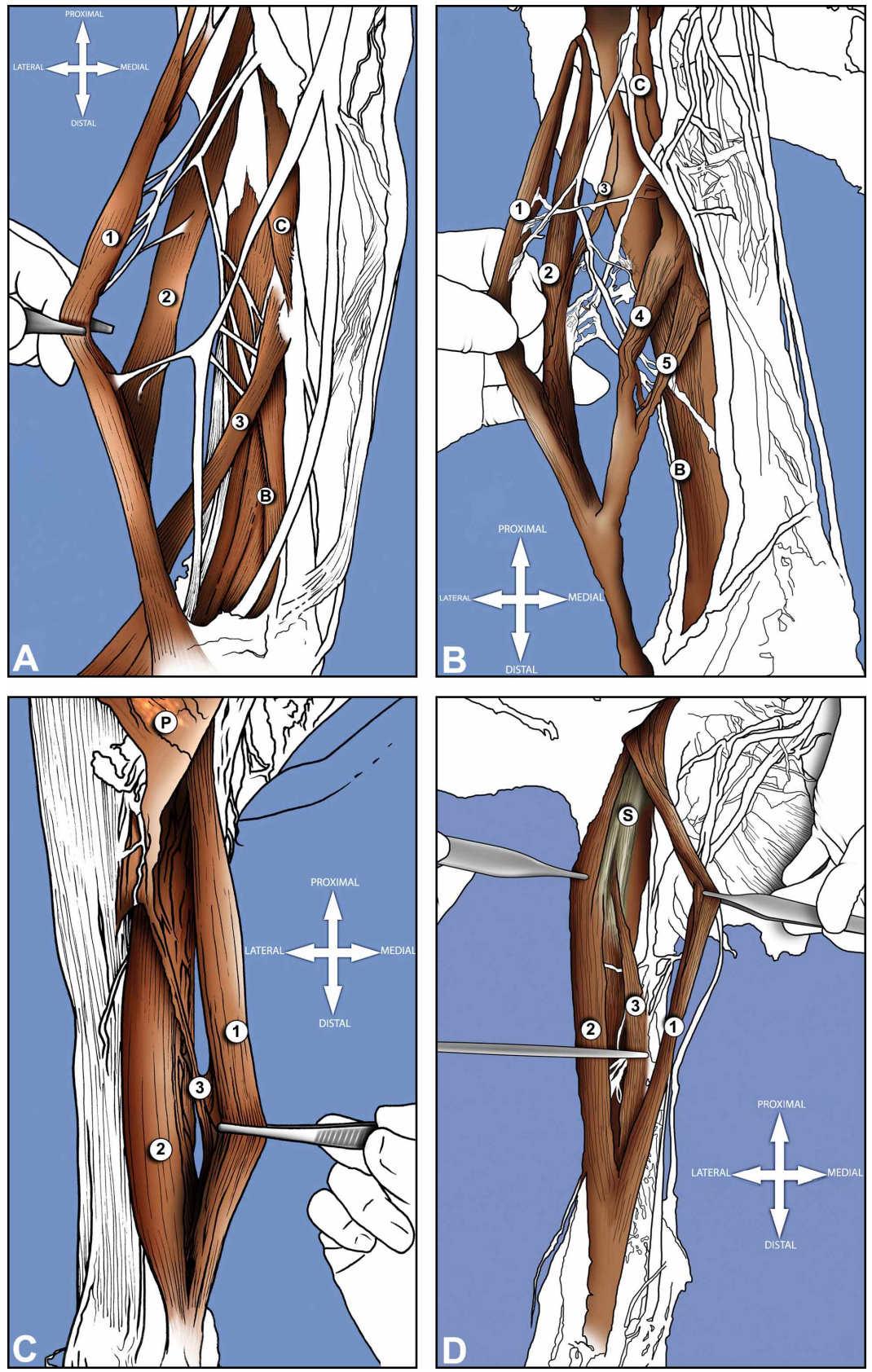

Fig. 1. Las figuras corresponden a la cara anterior del brazo derecho de cuatro diferentes cadáveres, donde aparece el músculo bíceps braquial (MBB) con sus correspondientes cabeza corta (1) y cabeza larga (2), además de diversas variaciones anatómicas, en las cuales se numeraron del 3 en adelante las cabezas accesorias que estaban presentes. La Fig. A muestra un MBB con el tipo de variación denominado I. 3b, porque su cabeza accesoria (3) se origina en la cara anteromedial del húmero, entre la inserción del músculo coracobraquial (C) y el origen del músculo braquial (B). La Fig. B muestra un MBB con una variación Tipo III, debido a la presencia de tres cabezas accesorias, una tipo 1e (3), con una pequeña cabeza accesoria originada en la cresta lateral del surco intertubercular, otra tipo 3b (4), similar a la encontrada en la Figura A y otra tipo 3d (5), con origen en el músculo braquial (B). La Fig. C muestra una variación Tipo I. 2b, porque la cabeza accesoria (3) se origina en el margen inferior del músculo pectoral mayor (P). La Fig. D muestra una variación Tipo I. 1e, porque la cabeza accesoria (3) se origina en la cresta lateral del surco intertubercular (S), de forma similar a como lo hace una de las cabezas accesorias de la Fig. B. Fuente: Autores.” las distintas regiones del brazo, así como la clase de tejido en que se origina, se crearon las clasificaciones por categoría en superior tejido óseo (1), superior tejido blando (2), medial (3) y lateral (4), ordenándolas de superior a inferior y de medial a lateral, en el caso de una variación donde estén implicadas dos cabezas supernumerarias o más. También fue creada la categoría de otras (5) para aquellas cabezas accesorias que no cumplen con los criterios acordados. Una tercera categorización fue creada basada en el origen real de la cabeza accesoria, denominándolo con las letras a, b, c, d, e, como se especifica en la Tabla I.

Dado que se contaba con un tamaño de muestra reducido, las estimaciones de las prevalencias y sus intervalos de confianza se hicieron considerando las cantidades de datos de cada variación presentes en la muestra. En los casos en los que la cantidad esperada de éxitos era menor a cinco, se utilizó el método exacto para hacer el cálculo, asumiendo una distribución binomial de probabilidades con probabilidad de éxito igual a la probabilidad de presentar la respectiva variación. Los datos fueron analizados mediante el programa estadístico SPSS, versión 22.0 @ .

\section{RESULTADOS}

De los 46 cadáveres estudiados, se encontró que $9(19,6 \%)$ presentaron variación en el MBB con respecto al patrón considerado normal, IC $95 \%$ (0,077-0,314), de los cuales $2(4,3 \%)$ lo hicieron bilateralmente y $7(15,2 \%)$ lo hicieron unilateralmente; $4(8,7$ $\%)$ derechos y $3(6,5 \%)$ izquierdos. Del total de 90 brazos estudiados, en $11(12,2 \%)$ los músculos presentaron cabezas accesorias o supernumerarias. Debido a que solo se contó con $2(4,3 \%)$ cadáveres femeninos, el sexo no pudo ser tenido en cuenta como variable.

Atendiendo al número de cabezas accesorias en los 11 brazos cuyos músculos presentaron la variación, $9(81,8 \%)$ fueron del Tipo I ( 1 cabeza accesoria), 1 (9,1\%) fue del Tipo II (2 cabezas accesorias) y 1 (9,1\%) 
fue del Tipo III (3 cabezas accesorias). El origen más frecuente de la cabeza accesoria entre las 14 cabezas supernumerarias encontradas fue el clasificado como 3 b (3 para la ubicación medial y b para el intervalo entre la inserción del músculo coracobraquial y el origen del músculo braquial) que se presentó en 11 (78,6 \%) músculos (Fig. 1A y Fig. 1B). El segundo lugar en frecuencia lo compartieron las que se originaban en el músculo braquial (clasificación 3d) (Fig. 1B), en el margen inferior del músculo pectoral mayor (clasificación 2b) (Fig. 1C) y en la cresta lateral del surco intertubercular del húmero (clasificación 1e) (Fig. 1D).

\section{DISCUSIÓN}

Se estima que la frecuencia con que se presentan las cabezas accesorias del MBB oscila en una rango amplio, que va desde 2,1\% reportado en India (Kumar et al., 2008) hasta 37,5\% reportado en Colombia (Rincón et al., 2002), siendo la encontrada en el presente estudio una de las más altas $(19,6 \%)$, en concordancia con lo reportado por Ballesteros et al. en su estudio realizado con 53 cadáveres en la ciudad de Bucaramanga, Colombia, en el cual reportan una frecuencia del 19,8\% para la presencia de cabezas accesorias del MBB.

De acuerdo con la clasificación propuesta, el tipo de cabeza accesoria hallada con mayor frecuencia fue la $3 \mathrm{~b}$ (la ubicada inferior y medialmente en el brazo y originada en el intervalo entre la inserción del músculo coracobraquial y el origen del músculo braquial), que se presentó en el 78,6 \% de los músculos accesorios encontrados, en concordancia con lo reportado en estudios previos (Asvat et al; Rincón et al.; Rodríguez-Niedenführ et al.; Ilayperuma et al.; Lee; Nasr \& Hussein, 2013). De acuerdo a la literatura revisada, no se encontró mayor diferencia en la lateralidad de la variación (Asvat et al.; Rodríguez-Niedenführ et al.), siendo la presentación más frecuente la unilateral derecha, lo cual también está acorde con otros reportes (Rodríguez-Niedenführ et al.; Ilayperuma et al.; Lee; Nasr \& Hussein).

Existe un reporte de caso en el cual se correlacionaron las variaciones encontradas en las salas de disección de anatomía con los síntomas reportados en la historia clínica del paciente, y se comprueba que en el miembro superior donde se observó una cabeza accesoria del MBB el paciente presentaba parestesias y disminución de la velocidad de electroconducción del nervio mediano, correlacionándose estos hallazgos con un canal formado por la cabeza accesoria del MBB sobre vasos y nervios del miembro superior afectado, interpretándose esto como la posible etiología de los síntomas del paciente (Paraskevas et al., 2008). En la clasificación propuesta, la cabeza 3c, aquella que se origina en el septo intermuscular medial, es la más propensa por su disposición a ser vinculada como posible factor etiológico de síndromes de compresión nerviosa, y aunque su frecuencia es menor que la de otras como la $3 \mathrm{~b}$, su relevancia como blanco terapéutico o quirúrgico hace importante que sea conocida por cirujanos y otros profesionales de la salud que tratan a pacientes con estas patologías.

Kervancioglu \& Orhan (2011) sugieren emplear las cabezas accesorias del MBB como colgajo para cubrir defectos en el brazo; sin embargo, no todas las cabezas presentan el volumen y el área necesarios para cubrir un defecto y raras veces se encuentran superficiales y fácilmente asequibles para el equipo quirúrgico, existiendo otras opciones más viables para estos procedimientos. Sin embargo, es relevante para las ciencias quirúrgicas el conocer de la existencia de cabezas accesorias del MBB, especialmente en regiones geográficas donde se ha reportado una alta prevalencia, para evitar errores durante procedimientos quirúrgicos al manipularlas mal (Warner et al.) y errores en el diagnóstico radiológico (Gheno et al.).

En un estudio previo realizado en Colombia por Rincón et al. con 16 cadáveres, se encontró la frecuencia de cabezas accesorias del MBB más alta reportada en la literatura. No obstante, la muestra estudiada fue relativamente pequeña, por lo que el presente estudio y el realizado por Ballesteros et al. ofrecen una muestra de la población colombiana de mayor tamaño, según la literatura revisada. Por la importancia que las variaciones anatómicas de distinta índole pueden tener en la práctica médica y afines, se sugiere que éstas sean incluidas en el contenido de los programas de anatomía de las distintas profesiones relacionadas con el cuidado de la salud.

Por la ausencia de una adecuada clasificación de las variaciones que puede presentar el MBB, se hace una propuesta para subsanar el faltante y se recomienda su utilización al momento de reportar estos casos. Así mismo, se encuentra una alta frecuencia de la variación del MBB en la muestra de la población colombiana estudiada la cual, para algunos tipos y subtipos de la clasificación propuesta por los autores, puede tener implicaciones clínicas, quirúrgicas y radiológicas relevantes.

AGRADECIMIENTOS. El presente trabajo fue exhibido en modalidad de ponencia oral en el XII Congreso Colombiano de Morfología, presentado el 30 de septiembre de 2016 en Cali, Colombia. Se agradece a las Universidades del Valle, Santiago de Cali y Libre - Seccional Cali, por permitir la realización del presente estudio en sus salas de disección. Agradecemos al señor Jorge Ernesto Ávila por el procesamiento y edición de las imágenes. 
GUERRERO, A.; BAENA-CALDAS, G. P.; PEÑA, E.; TOVAR, C. J. R. \& CORRALES, L. M. Proposal of classification for the variations of the biceps brachii muscle and study in a sample of Colombian population. Int. J. Morphol., 35(1):54-58, 2018.

SUMMARY: The anatomical variations of the biceps brachii muscle in relation to the number of supernumerary or accessory bellies may have important incidence in clinical and radiological diagnoses, as well as in surgical procedures. The absence of an appropriate classification for the variations that the biceps brachii muscle can present and the findings of the present study, allowed the authors to present a classification proposal based on the number of accessory bellies and their origin. The study aimed to determine with which frequency the variations in the number of accessory bellies of the biceps brachii muscle are present, and to propose a classification for these variations. In the crosssectional, descriptive study 90 arms of 46 Colombian cadavers were analyzed. A high frequency (19.6\%) in the variations of the muscle was found, $4.3 \%$ bilaterally and $15.2 \%$ unilaterally. In the 11 arms $(12.2 \%)$ that presented the variation, the presence of only one accessory belly was the highest frequency $(81.8 \%)$, being the most frequent origin for them the one classified by the authors as $3 \mathrm{~b}, 3$ for the inferomedial situation and $\mathrm{b}$ for the interval between the insertion of the coracobrachii muscle and the origin of the brachii muscle. Because the classification proposed is very detailed, its use is recommended whenever cases of biceps brachii muscle variations are reported. In the cadavers of the Colombian population studied, a high frequency was found for the presence of supernumerary or accessory heads of this muscle, which deserves to be known by medical professionals.

KEY WORDS: Anatomic variation; Biceps brachii muscle; Accessory bellies; Arms; Cadaver.

\section{REFERENCIAS BIBLIOGRÁFICAS}

Asvat, R.; Candler, P. \& Sarmiento, E. E. High incidence of the third head of biceps brachii in South African populations. J. Anat., 182(Pt. 1):1014, 1993.

Ballesteros, L. E.; Forero, P. L. \& Buitrago, E. R. Evaluation of additional head of biceps brachii: a study with autopsy material. Folia Morphol. (Warsz.), 73(2):193-8, 2014.

Bergman, R. A.; Thompson, S. A. \& Afifi, A. K. Compendium of Human Anatomic Variation: Text, Atlas, and World Literature. Baltimore, Urban \& Schwarzenberg, 1988.

Delgado Garcia, A. Anatomía Humana Funcional y Clínica. Cali, Universidad del Valle, 1996

Duran, J. T. C. \& Arquez, H. F. A third yead of the biceps brachii: An anatomical insight. J. Chem. Pharm. Res., 8(8):669-74, 2016.

Gheno, R.; Zoner, C. S.; Buck, F. M.; Nico, M. A.; Haghighi, P.; Trudell, D. J. \& Resnick, D. Accessory head of biceps brachii muscle: anatomy, histology, and MRI in cadavers. A. J. R. Am. J. Roentgenol., 194(1):W80-3, 2010.

Ilayperuma, I.; Nanayakkara, G.\& Palahepitiya, N. Incidence of humeral head of biceps brachii muscle: Anatomical insight. Int. J. Morphol., 29(1):221-5, 2011.
Kervancioglu, P. \& Orhan, M. An anatomical study on the three-headed biceps brachii in human foetuses, and clinical relevance. Folia Morphol. (Warsz.), 70(2):116-20, 2011.

Kumar, H.; Das, S. \& Rath, G. An anatomical insight into the third head of biceps brachii muscle. Bratisl. Lek. Listy., 109(2):76-8, 2008.

Lee, J. H. The third head of biceps brachii muscle in korean: Anatomical study. Korean J. Phys. Anthropol., 26(3):97-100, 2013.

Lutterbach-Penna, R. A.; Brigido, M. K.; Robertson, B.; Kim, S.M.; Jacobson, J.A. \& Fessell, D.P. Sonography of the accessory head of the biceps brachii. J. Ultrasound Med., 33(10):1851-4, 2014.

Nakatani, T.; Tanaka, S. \& Mizukami, S. Bilateral four-headed biceps brachii muscles: the median nerve and brachial artery passing through a tunnel formed by a muscle slip from the accessory head. Clin. Anat., 11(3):20912, 1998.

Nasr, A. Y. \& Hussein, A. M. Morphology and clinical implication of the extra-head of biceps brachii muscle. Folia Morphol. (Warsz.), 72(4):349-56, 2013

Nayak, S. R.; Krishnamurthy, A.; Kumar, M.; Prabhu, L. V.; Saralaya, V. \& Thomas, M. M. Four-headed biceps and triceps brachii muscles, with neurovascular variation. Anat. Sci. Int., 83(2):107-11, 2008.

Paraskevas, G.; Natsis, K.; Ioannidis, O.; Papaziogas, B.; Kitsoulis, P. \& Spanidou, S. Accessory muscles in the lower part of the anterior compartment of the arm that may entrap neurovascular elements. Clin. Anat., 21(3):246-51, 2008.

Rincón, F.; Rodríguez, Z. I.; Sánchez, A.; León, A. \& González, L. F. The anatomic characteristics of the third head of biceps brachii muscle in a Colombian population. Rev. Chil. Anat., 20(2):197-200, 2002.

Rodríguez-Niedenführ, M.; Vázquez, T.; Choi, D.; Parkin, I. \& Sañudo, J. R. Supernumerary humeral heads of the biceps brachii muscle revisited. Clin. Anat., 16(3):197-203, 2003.

Warner, J. J.; Paletta, G. A. \& Warren, R. F. Accessory head of the biceps brachii. Case report demonstrating clinical relevance. Clin. Orthop. Relat. Res., (280):179-81, 1992.

\author{
Dirección para correspondencia: \\ Alian Guerrero \\ Médico y Cirujano \\ Estudiante de Maestría en Ciencias Biomédicas \\ Facultad de Salud \\ Universidad del Valle \\ Cali \\ COLOMBIA
}

Email: alian.guerrero@correounivalle.edu.co

Recibido : 11-04-2017

Aceptado: 28-09-2017 